\title{
Economic impact payment, human mobility and COVID-19 mitigation in the USA
}

\author{
Ruohao Zhang ${ }^{1,2}$ (D
}

Received: 20 August 2020 / Accepted: 9 August 2021 / Published online: 20 August 2021

(c) The Author(s), under exclusive licence to Springer-Verlag GmbH Germany, part of Springer Nature 2021

\begin{abstract}
This paper studies the effect of the economic impact payment (EIP) on individual contributions to COVID-19 mitigation efforts in the USA, where the mitigation efforts are measured by the reduction of daily human mobility. I empirically estimate the effect of the EIP in April 2020 and use cellphone GPS data of 45 million smartphone devices as a proxy for human mobility across 216,069 Census Block Groups. The results show that when receiving the EIP, households significantly increased "Median Home Dwell Time" by an average of 3-5\% (about 26-45 $\mathrm{min}$ ). The paper highlights this unintended effect of the EIP, namely, that in addition to providing economic assistance, the EIP also helped increase individual contributions to mitigation efforts that slowed COVID-19 virus transmission in early 2020.
\end{abstract}

Keywords Public good - Economic impact payment - COVID-19 · Mitigation effort · Human mobility $\cdot$ Income effect

JEL Classification D11 · D12 · H41 · I12 · I14 · I18

\section{Introduction}

In the absence of medical countermeasures, non-pharmaceutical interventions (NPI) have become a primary policy tool to mitigate the spread of COVID-19 virus during the pandemic of 2020. Under NPI policies, the public is expected to contribute to

I would like to thank Neha Khanna, Solomon Polachek and David Slichter from Binghamton University
and Huan Li from North Carolina A\&T State University for helpful comments. I would like to thank the
Editor-in-Chief Arthur H. O. van Soest, the anonymous associate editor and two anonymous referees of
Empirical Economics for their constructive comments. I alone am responsible for any remaining errors.

$凶$ Ruohao Zhang

ruohao.zhang@law.northwestern.edu

1 Kellogg School of Management, Northwestern University, Evanston, IL, USA

2 Pritzker School of Law, Northwestern University, Evanston, IL, USA 
mitigation efforts through behavioral modifications, such as social distancing, wearing masks and following shelter-in-place and quarantine requirements. Although the mitigation efforts of uninfected people only protect themselves from infection, those made by infected people can help protect others. In the early stage of the COVID-19 pandemic, the vast majority of the population was unaware of their infection status, because COVID-19 testing resources were not yet widely available and COVID-19 symptoms, which are similar to influenza, were not easy to diagnose without testing. This lack of awareness created an incentive for all individuals, regardless of their infection status, to contribute to mitigation efforts to help slow the spread of the virus. Such social mitigation efforts can be treated as a public good.

Due to the nature of public goods, market-determined individual contributions to COVID-19 mitigation efforts are always lower than the socially optimal level. Government interventions help increase the social aggregate mitigation efforts and raise the associated social welfare. A typical instrument of government interventions is to impose mandatory policies, which can raise the lower bounds of all individual contributions to mitigation efforts. If properly enforced, mandatory policies can be very effective in raising social aggregate mitigation efforts. However, social and political factors can make it difficult to enforce mandatory policies. In this paper, I argue that in addition to mandatory policies, a useful alternative policy instrument is to increase social aggregate mitigation efforts using income compensation. According to the theory of public goods, if the income compensation is properly customized according to each individual's income and to the status quo of the social aggregate mitigation efforts, it can also be effective in increasing individual mitigation effort contributions.

For this study, I construct a data set of human mobility to empirically test the argument that income compensation can raise contributions to COVID-19 mitigation efforts through the decrease in human mobility, an important mitigation factor in the early stages of the COVID-19 pandemic. In the data set, human mobility is measured by daily home dwell time. I utilize the first round of economic impact payment (EIP) in April 2020 as a quasi-natural experiment. EIP was an exogenous government payment to individuals/households in the USA to provide economic assistance during the COVID-19 pandemic. It provided an opportunity to test whether receiving this income compensation led to a decrease in the amount of individual mobility. The empirical results show that the EIP significantly increased individual home dwell time by an average of 3 to 5\% (about 26-45 min) per day. Among all income groups, only the mobility of the households with income less than $\$ 20,000$ appeared to be unaffected by the EIP, because the social aggregate mitigation efforts before the EIP were already higher than the optimal mitigation efforts of these households, and the amount of the EIP was not large enough to raise their optimal mitigation efforts above the social aggregate level, so they had no incentive to contribute additional mitigation efforts even after receiving the EIP. For all other income groups, receiving the EIP increased their daily home dwell time, suggesting that the EIP motivated them to increase their contributions to mitigation efforts. In addition, the results also suggest that mandatory mitigation policies were not strictly enforced in the USA, so the government was unable to make meaningful mandatory mitigation requirements for controlling COVID-19. 
This paper contributes to three bodies of the literature. Firstly, the public goods literature has illustrated the relationship between income and the contributions to public goods (Buckley and Croson 2006; Kotchen and Moore 2007; Jacobsen et al. 2012). I expand this literature by incorporating the public goods model developed in Kotchen and Moore (2007) with government mandatory policies. In addition, I derive the heterogeneous changes of contributions to public goods in response to income compensation for individuals from different income groups. Secondly, the empirical literature on the EIP and other similar stimulus payments during past crises in the United States, such as the 2001 tax rebate and the 2008 stimulus payment, has shown that stimulus payments significantly increased households' private consumption (Johnson et al. 2006). Such stimulus payments, however, also have adverse health impacts, possibly because of the increase in alcohol and drug consumption (Evans and Moore 2011; Gross and Tobacman 2014). This paper contributes to the literature on the effects of the EIP by focusing on a different consumption category, namely public goods. Third, and most importantly, this work adds to the current and rapidly evolving literature on COVID-19, specifically to the category of papers that studied the effects of government policies on human mobility during the pandemic (Dave et al. 2020; Gupta et al. 2020; Wright et al. 2020), the relationship between human mobility and the spread of the coronavirus (Farboodi et al. 2021; Chudik et al. 2020; Fowler et al. 2021; Kapoor et al. 2020) and the effectiveness of the government mitigation policies on controlling the spread of COVID-19 (Gollwitzer et al. 2020; Allcott et al. 2020; Wright et al. 2020). However, to the best of my knowledge, this is the only paper that studies the public goods nature of COVID-19 mitigation efforts and infers a possible decentralized solution to help mitigate the spread of virus. This paper suggests that in addition to its primary purpose of easing immediate economic strife, the EIP also motivates individuals to increase contributions to mitigation efforts.

\section{Background and testable hypothesis}

\subsection{Theoretical background}

In the context of a global infectious disease outbreak, mitigation efforts can be treated as consumable goods because they bring private utility gains to individuals by reducing infection risk. Contributing to mitigation efforts also yields private costs, however, including reduced working hours, added expenses for personal protection equipment, restricted travel plans, cancelled entertainment events and so on. Yet mitigation efforts can be treated as a public good overall because they reduce both the rate of virus transmission and the infection risk for the public as a whole.

Seen as a public good, mitigation efforts are by definition non-rival and nonexcludable. Each individual determines her own optimal mitigation efforts based on both income and an assessment of other individuals' contributions to mitigation efforts. In order to illustrate individual mitigation effort contributions at the social equilibrium, I construct a theoretical model following the setting of Kotchen and Moore (2007). The model implies that at the market-determined social equilibrium, social aggregate mitigation efforts are either greater than or equal to every individual's optimal levels. 
This model separates all the individuals into two types: "voluntary contributors" and "non-voluntary contributors." For the individuals whose optimal mitigation efforts are equal to the social aggregate level, they are defined as "voluntary contributors," because their optimal mitigation efforts are higher than the aggregation of all other individuals' contributions. Therefore, they have the incentive to contribute additional mitigation efforts to raise the social aggregate level to the equilibrium. For the individuals whose optimal mitigation efforts are less than the social aggregate level, they are defined as "non-voluntary contributors," because their optimal mitigation efforts are less than the aggregation of all other individuals' contributions. Therefore, "nonvoluntary contributors" have no incentive to raise the social aggregate level and do not contribute to mitigation efforts. Given the assumption that mitigation efforts are normal goods, individual's optimal mitigation efforts increase with incomes. ${ }^{1}$ According to the status quo of social aggregate mitigation efforts at the equilibrium, there exists an income threshold level that determines whether an individual is a "voluntary contributor" or "non-voluntary contributor" to mitigation efforts. The detail of the theoretical model is described in Online Appendix A.1.

With positive externalities, the market-determined social equilibrium is always lower than the socially optimal solution, suggesting that government interventions are needed to increase the social aggregate mitigation efforts. One commonly used instrument in government interventions is mandatory policies. With mandatory policies, individual mitigation efforts consist of two parts: the mandatorily required mitigation efforts and the additional voluntary mitigation efforts. The mandatorily required mitigation efforts are non-negative and serve as lower bounds of individual mitigation efforts. The individuals who are "non-voluntary contributors" only contribute at the mandatory level, whereas the individuals who are "voluntary contributors" contribute additional voluntary mitigation efforts other than the mandatory requirement (see Propositions 1 and 2 in Online Appendix A.1).

In addition to mandatory policies, another less well-known instrument of government intervention is income or monetary compensation. Income compensation may be effective in increasing social aggregate mitigation efforts in two ways. For individuals who are "voluntary contributors," recall that their incomes are above the income threshold, and they make positive additional voluntary mitigation efforts. Accordingly, a marginal income compensation can increase their additional voluntary mitigation efforts. However, for the individuals who are "non-voluntary contributors," their mitigation efforts always remain at the mandatory level as long as their incomes are below the income threshold. A marginal income compensation does not affect their contributions to mitigation efforts. This suggests that the amount of income compensation must be large enough to raise this group's incomes above the income threshold in order to convert them into "voluntary contributors."

This theory has important policy implications: Although the government can increase the social aggregate mitigation efforts by imposing or increasing the stringency of existing mandatory policies, there may be social or political factors that make

\footnotetext{
${ }^{1}$ It is reasonable to assume that COVID-19 mitigation efforts are normal goods since they are health-related. Literature usually finds the income elasticity of health expenditure positive. For example, Acemoglu et al. (2013) found that income elasticity of health expenditure equaled 0.7 for the USA from 1960 to 2005 . So it is reasonable to assume that individual expenditure of health-related goods increases with income.
} 
them difficult to enforce. As an alternative, income compensation, if properly distributed, can be a good complement to mandatory policies. To be effective, the income compensation should be carefully customized for "non-voluntary contributors" with income lower than the income threshold, so that it can help raise this group's incomes sufficiently to increase its mitigation effort contributions.

\subsection{Empirical background}

On March 27, 2020, the Coronavirus Aid, Relief, and Economic Security (CARES) Act was passed by the US Congress and signed into law by President Trump. The CARES Act contained a $\$ 2$ trillion economic relief package that aimed to assist households, small businesses, healthcare system and local governments. ${ }^{2}$ A portion of the $\$ 2$ trillion package was used as the first wave of an economic impact payment (EIP) providing economic assistance to individuals/households during the pandemic. An eligible taxpayer, who was single and had an annual income of less than $\$ 75,000$, or a head of a household with an annual income under $\$ 112,500$ received an EIP of $\$ 1,200$. Eligible married couples that filed taxes jointly with annual incomes less than $\$ 150,000$ received an EIP of $\$ 2400$. Households with children under 17 received an additional $\$ 500$ per child for up to two children. Some taxpayers with higher annual incomes also received a reduced EIP. The first wave of EIP distribution was made by the IRS beginning the week of April 13, 2020, and was paid through direct deposits, followed by the subsequent paper check distribution. ${ }^{3}$ Although the primary purpose of the EIP was to provide economic relief, this lump-sum payment also exogenously increased recipients' incomes, making it possible to be used to test the effect of income compensation on individual contributions to COVID-19 mitigation efforts.

Recall that individual contributions to COVID-19 mitigation efforts consist of two components: the mandatory mitigation efforts and the additional voluntary mitigation efforts. Mandatory contributions are minimum contributions, strictly enforced by the government. If there is a policy that is mandated but not effectively enforced and relies solely on self-compliance, then this associated mitigation efforts should be considered as additional voluntary mitigation efforts, since the levels of compliance are mostly voluntarily determined. In the USA, most of the COVID-19 NPIs were not strictly enforced and relied heavily on self-compliance, which makes it difficult to determine whether or not it was a mandatory mitigation requirement that was meaningful. Several papers that examined the effects of NPI provided strong evidence that mitigation efforts in the USA had been largely voluntary. For example, Wright et al. (2020) found that under the shelter-in-place policy, the people in high-income counties significantly reduced social mobility, whereas mobility remained at the same levels in low-income counties. Compliance with NPIs also depended substantially on individual characteristics, including partisanship and conservative media exposure (Allcott et al. 2020;

\footnotetext{
2 https://home.treasury.gov/policy-issues/cares.

3 The IRS announced on Twitter that the first EIP deposit would occur on April 11, 2020. Given that this was a Friday, most EIP direct deposits would not arrive until the week of April 13. The timeline for EIP distribution published by the House Ways and Means Committee also indicated that EIP direct deposit would be during the week of April 13 (https://twitter.com/IRSnews/status/1249062356077944832).
} 
Gollwitzer et al. 2020), social capital (Barrios et al. 2021), levels of trust in the media, science and government (Bai et al. 2020; Barrios et al. 2021; Durante et al. 2021) and so on. Similar empirical results are found in this paper, indicating that COVID-19 NPIs are not effectively enforced as mandatory policies in the USA.

\subsection{Hypothesis}

As a form of income compensation, an EIP may affect social aggregate contributions to mitigation efforts. In order to increase individuals' contributions to mitigation efforts, those who receive an EIP must have incomes greater than the income threshold (determined by the status quo social aggregate level at the equilibrium) subtracted by the amount of the EIP. These individuals are either "voluntary contributors" (if their incomes are greater than the income threshold) who will increase their mitigation efforts in response to a marginal increase in incomes, or they are "non-voluntary contributors" (if their incomes are smaller than the income threshold but greater than the income threshold minus the amount of EIP) so that they are converted into "voluntary contributors" after receiving the EIP. In both cases, the EIP increases these individuals' contributions to mitigation efforts, which leads to higher social aggregate mitigation efforts.

In the empirical analysis, I use human mobility at the census block group (CBG) level as an indicator of COVID-19 mitigation efforts. By assuming that in most CBGs, there are individuals who not only are eligible to receive the EIP but who also increase their mitigation efforts in response, we have the following hypothesis:

Main hypothesis The aggregate human mobility in the CBG decreases in response to receiving EIP. ${ }^{4}$

\section{Data}

To empirically test the main hypothesis that the EIP causes a decrease in human mobility, I assemble a CBG-by-day data set that covers 216,069 CBGs across 50 states and Washington, DC, in the USA (including Alaska and Hawaii). The sample period is between April 1, 2020, and April 20, 2020. The data set includes information on household income distribution and daily human mobility at the CBG level, daily COVID-19 confirmed case and death numbers at the county level, and government NPI policies at both the state and county levels. ${ }^{5}$

I obtain human mobility data from the "Social Distancing Metrics" database provided by SafeGraph, Inc. The data are collected from GPS locations of 45 million smartphone devices in the USA and are aggregated at the CBG level. I use "Median Home Dwell Time" as the outcome variable to measure human mobility. "Median Home Dwell Time" calculates the median number of minutes that smart phone devices

\footnotetext{
4 As another potentially testable implication, a higher mandatory requirement should increase the social aggregate mitigation efforts, mainly driven by lower-income individuals. In this paper, I do not focus on testing this hypothesis, because COVID-19 NPIs in the USA are largely depending on self-compliance. See empirical evidence in Appendix Tables B1 and B5.

5 I use household incomes because Census data does not report individual income distribution.
} 
stay at home throughout a single day. "Home" is identified as an $153 m \times 153 m$ square in which the smart phone devices are mostly located during the overnight hours between 6:00 PM and 7:00 AM. "Home" location is updated at the beginning of each month, according to the previous 6-week overnight hours locations. SafeGraph calculates a "confidence score" for the cellphones' overnight hours locations. Devices with low confidence scores are treated as if the home location is unknown. ${ }^{6}$ One concern of these data is that the "Home" location may be misspecified for college students who returned back home when the schools were closed due to the pandemic. Since the analysis is limited within the month of April, all cellphones in the sample had their "Home" location updated at April 1, 2020, whereas school closures took place earlier in mid-March (Mangrum and Niekamp 2020). Therefore, the college students who left the campus are treated as having unknown home location, so they are not included in the data and therefore do not affect the analysis.

The county-level COVID-19 confirmed cases and death data (JHU data) are collected by the Center for Systems Science and Engineering at John Hopkins University. JHU data are publicly available, and it covers the COVID-19 pandemic in the USA from January 22, 2020 (the first case identified in the USA), to the present. The statelevel and county-level government NPI policy data (Keystone data) are obtained from Keystone Strategy. ${ }^{7}$ Keystone data collect information from government Web sites and local news reports and are updated on April 16, 2020. Keystone data provide statelevel policy data for all states, and county-level policy data for all counties with at least 100 confirmed cases as of April 6, 2020. I consider the following three of the state and county policies reported by Keystone data that had the most significant effect on human mobility: "Social Distancing," "Non-essential Services Closure" and "Shelterin-Place." "Social Distancing" was a requirement that people maintained physical separation in public space. It reduced the number of times that people physically made contact or came close to one another. "Non-essential Services Closure" paused all economic activity that was not essential to sustain health and safety. "Shelter-inPlace" was a more general and the strictest order that required people to minimize their social activities. ${ }^{8}$

Census data provide detailed information about CBG characteristics. Since the census data do not provide time variation on a daily basis, most of the characteristics are captured by the CBG fixed effect. However, variations in incomes are important to be considered for two reasons. First, it identifies the percentage of households receiving the EIP. Second, the EIP effect on human mobility is hypothesized to be different across income groups. EIP eligibility is based on 2018 or 2019 annual incomes in federal tax filings. The Census Bureau only reports incomes for household units, and 2019

\footnotetext{
6 "Confidence score" is calculated by the number of unique hours and unique days for which GPS pings were observed at the "Home" location. See https://docs.safegraph.com/docs/places-manual\#section-safegraph-common-nighttime-location-algorithm.

7 US government NPI policy data are collected by Keystone Strategy, partnered with Susan Athey, Stanford Professor of Economics, and Marco Iansiti, Director of Harvard Business School's Digital Initiative. See https://www.keystonestrategy.com/coronavirus-covid19-intervention-dataset-model.

8 Since "Non-essential Services Closure" is always included within the "Shelter-in-Place" order, I assume that the "Non-essential Services Closure" order is substituted by the "Shelter-in-Place" order once the latter is issued.
} 


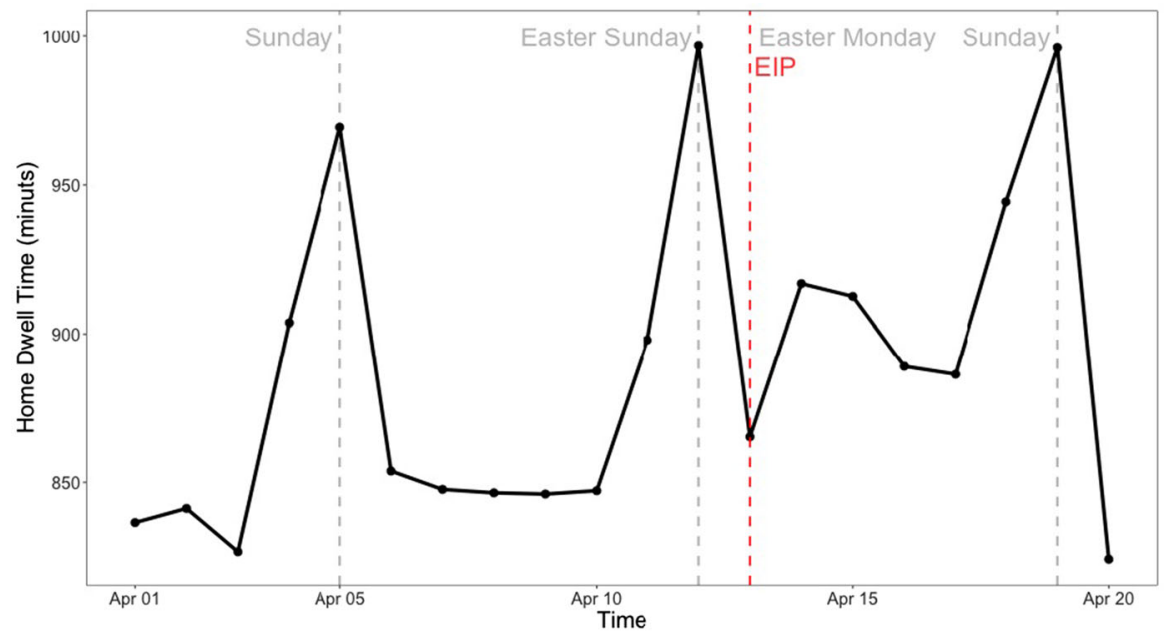

Fig. 1 Daily average Median Home Dwell Time

income data at the CBG level are not available by the time of this study, so I use census 2014-2018 5-year estimates household income data to proxy current income distribution. ${ }^{9}$

Table 1 reports the summary statistics of the data set. The table's first panel reports "Median Home Dwell Time," the percentage of households in each income group and county COVID-19 statistics. On average, "Median Home Dwell Time" is 886 minutes (around 15 hours) per day. About $87.59 \%$ of all the households in each CBG have an income lower than $\$ 150,000$. The second panel reports state and county policies. It shows that only 13 states issued "Non-essential Services Closure" orders, because most of the states skipped this policy and directly issued the stricter "Shelter-in-Place" orders. These 13 states either issued "Shelter-in-Place" orders after first imposing a "Non-essential Services Closure" order, or they have not formally issued "Shelter-inPlace" order yet. County-level policies can be different from state-level policies since many county governments move faster than the state and set more stringent policies. ${ }^{10}$

Figure 1 shows the unconditional average daily trend of "Median Home Dwell Time" across all CBGs in the sample. As expected, in general "Median Home Dwell Time" is lower during the weekdays and higher during the weekends. Compared across weeks, the figure shows that for the week when the EIP is received, "Median Home Dwell Time" became much higher, suggesting people on average increased their time of staying at home through that week.

\footnotetext{
92019 income data at the CBG level for 2019 are not yet available as of May 2020, the time when this research is completed.

10 For example, New York State governor avoided using "Shelter-in-Place," so in the data, New York state did not issue a "Shelter-in-Place" order, but only an order mandating "Non-essential Services Closure." However, all the counties in New York City issued a "Shelter-in-Place" order.
} 
Table 1 Descriptive statistics

\begin{tabular}{|c|c|}
\hline $\begin{array}{l}\text { Panel I } \\
\text { Variable }\end{array}$ & Mean (standard deviation) \\
\hline Median Home Dwell Time (min) & $\begin{array}{l}886.6570 \\
(333.4201)\end{array}$ \\
\hline Income $(\$ 0-20,000)$ & $\begin{array}{l}0.1663 \\
(0.1371)\end{array}$ \\
\hline Income $(\$ 20,000-40,000)$ & $\begin{array}{l}0.1908 \\
(0.1098)\end{array}$ \\
\hline Income $(\$ 40,000-60,000)$ & $\begin{array}{l}0.1608 \\
(0.0864)\end{array}$ \\
\hline Income $(\$ 60,000-100,000)$ & $\begin{array}{l}0.2191 \\
(0.1009)\end{array}$ \\
\hline Income $(\$ 100,000-150,000)$ & $\begin{array}{l}0.1390 \\
(0.0944)\end{array}$ \\
\hline Income $(\$ 0-150,000)$ & $\begin{array}{l}0.8759 \\
(0.1449)\end{array}$ \\
\hline Income $(>\$ 150,000)$ & $\begin{array}{l}0.1241 \\
(0.1449)\end{array}$ \\
\hline County COVID-19 confirmed case number & $\begin{array}{l}2033.2778 \\
(7689.8043)\end{array}$ \\
\hline County COVID-19 death number & $\begin{array}{l}101.4488 \\
(736.4111)\end{array}$ \\
\hline \multicolumn{2}{|l|}{ Panel II } \\
\hline $\begin{array}{l}\text { Non-pharmaceutical interventions } \\
\text { (By April 20, 2020) }\end{array}$ & $\begin{array}{l}\text { Block group number } \\
\text { (state/county number) }\end{array}$ \\
\hline Social distancing (state) & $\begin{array}{l}183,637 \\
(40)\end{array}$ \\
\hline Social distancing (county) & $\begin{array}{l}116,063 \\
(291)\end{array}$ \\
\hline Non-essential services closure (state) & $\begin{array}{l}53,959 \\
(13)\end{array}$ \\
\hline Non-essential services closure (county) & $\begin{array}{l}124,606 \\
(322)\end{array}$ \\
\hline Shelter-in-Place (state) & $\begin{array}{l}174,272 \\
(42)\end{array}$ \\
\hline Shelter-in-place (county) & $\begin{array}{l}116,885 \\
(299)\end{array}$ \\
\hline \multicolumn{2}{|l|}{ Panel III } \\
\hline Number of census block groups & 216,069 \\
\hline Number of observations & $4,317,568$ \\
\hline
\end{tabular}




\section{Empirical analysis}

\subsection{Identification strategies}

I employ an event study design as the main empirical analysis, where the outcome variable is CBG-level human mobility that measures COVID-19 mitigation efforts, and the EIP is an exogenous treatment. According to the hypothesis that the EIP decreased human mobility, I expect the EIP to increase mitigation efforts since it compensated for households' incomes. One major challenge of this analysis is that the EIP is a (near) universal treatment for all CBGs across the USA, so we do not have a control group of CBGs that were not treated by the EIP. This is because in almost all CBGs there were always some households that were eligible for and had received the EIP. However, the size of the EIP treatment on each CBG was different, depending on the ratio of eligible households. I utilize the variation in the size of the EIP treatment across CBGs to identify the treatment effect on human mobility through the event study analysis, which compares two groups of CBGs that received different sizes of the EIP treatment. The causal effect of receiving the EIP can be identified if the event study finds a gap in the treatment effects between two groups.

In the event study, I set a cutoff of the ratio of households with an annual income of less than $\$ 150,000$ (who were therefore eligible for receiving the EIP) and use the cutoff to separate all CBGs into two groups: the high-income CBGs and the lowincome CBGs. ${ }^{11}$ For the high-income CBGs, the ratio of EIP-eligible households is below the cutoff; for the low-income CBGs, the ratio of EIP-eligible households is above the cutoff. According to this setting, the low-income $\mathrm{CBG}$ seceived a relatively greater size of the EIP treatment than the high-income CBGs. The event study was able to detect the EIP treatment effect by estimating the differences in human mobility between the low-income and high-income CBGs on a daily basis and comparing these daily differences between pre-treatment and post-treatment periods. According to the main hypothesis that the EIP reduced human mobility, I expect to observe significant changes in the daily differences after the EIP treatment was implemented. I set the cutoff at $75 \%$ and compare both the unconditional and conditional daily trend of "Median Home Dwell Time" between two groups of CBGs. The daily trend remains similar for different cutoff values between $60 \%$ and $80 \%$.

In addition, I also consider a difference-in-differences (DID) model with continuous treatment in online appendix as a supplement and robustness check for the event study analysis. The DID with continuous treatment is an extension of the classic binary potential outcome framework, which identifies the average treatment effect by allowing a series of potential outcomes with continuous variation in treatment levels. The detailed setting of the DID analysis is given in Online Appendix A.5.

I conduct all the analysis using both state-level and county-level policy data. Compared with the analysis using the state-level policy data, the county-level policy data capture more precisely the potential mandatory mitigation requirement. In addition, it

\footnotetext{
11 Although households with income higher than $\$ 150,000$ may receive reduced amount of EIP, I exclude them because relative to their incomes, a small lump-sum payment may yield very limited marginal change in their behavior.
} 


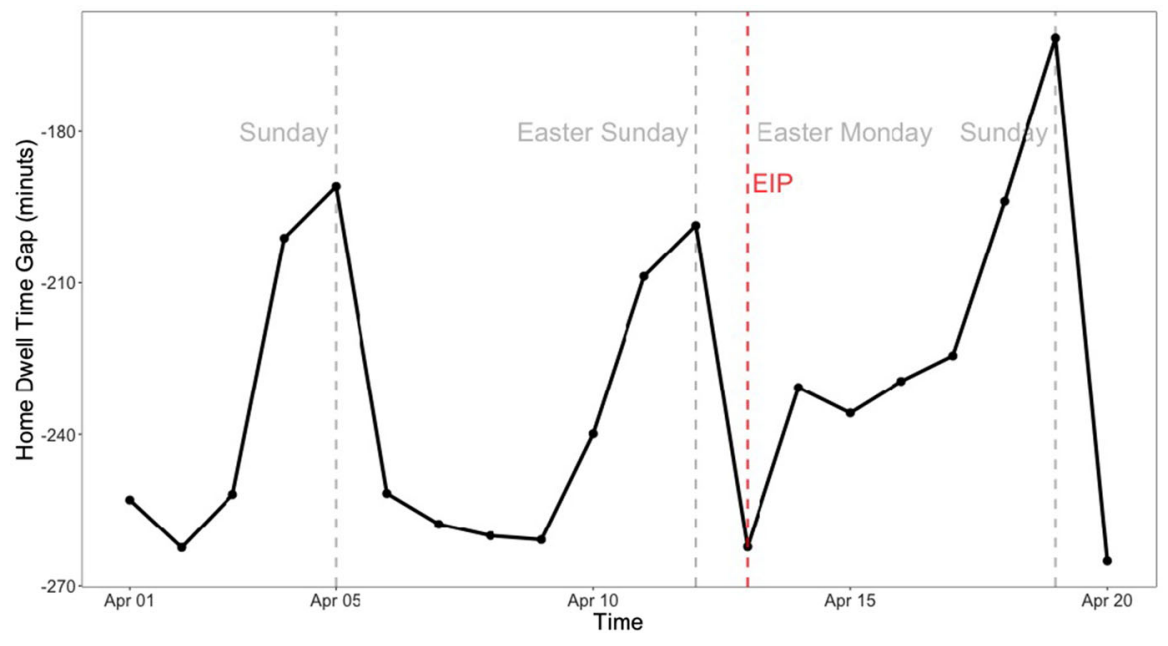

Fig. 2 Daily Median Home Dwell Time gap between CBGs with share of EIP reception more/less than $75 \%$

also allows me to test for different EIP treatment effects in the outbreak counties with more than 100 confirmed cases during the early phase of the pandemic.

\subsection{Event study}

I start the analysis by comparing the unconditional daily trend of "Median Home Dwell Time" between the low-income and high-income CBGs. Figure 2 shows the unconditional average daily gap of "Median Home Dwell Time" between the two groups, where the reference group is the high-income CBGs. As we expected, the gap is always negative, suggesting that the low-income CBGs always have lower "Median Home Dwell Time" than the high-income CBGs. It is worth mentioning that although the daily trend in the gap shows some pre-existing weekday versus weekend variation, after receiving the EIP, this gap decreases dramatically for both weekdays and weekends. Similar results are also shown in Fig. 3, where the unconditional time trends for high-income (EIP eligible rate below 75\%) and low-income (EIP eligible rate above $75 \%$ ) $\mathrm{CBG}$ are plotted.

Next, I compare the conditional time daily trends of "Median Home Dwell Time" for the low-income and high-income CBGs, using the following regression:

$$
\begin{aligned}
y_{i t}= & \sum_{a \in T} \theta_{a}^{1} I_{t=a} \times I_{c_{i}>75 \%}+\sum_{a \in T} \theta_{a}^{2} I_{e_{i t}=a} \times I_{c_{i} \leq 75 \%} \\
& +\beta x_{i t}+\rho z_{i t}+\lambda d_{t} \times I_{c_{i}>75 \%}+\mu_{i}+\epsilon_{i t},
\end{aligned}
$$

where $i$ is CBG index, $t$ is date index, $y_{i t}$ is CBG's human mobility and $x_{i t}$ is the covariates which capture the time-varying characteristics that are correlated with human mobility. I define $x_{i t}$ to be the number of COVID-19 confirmed cases and deaths at 


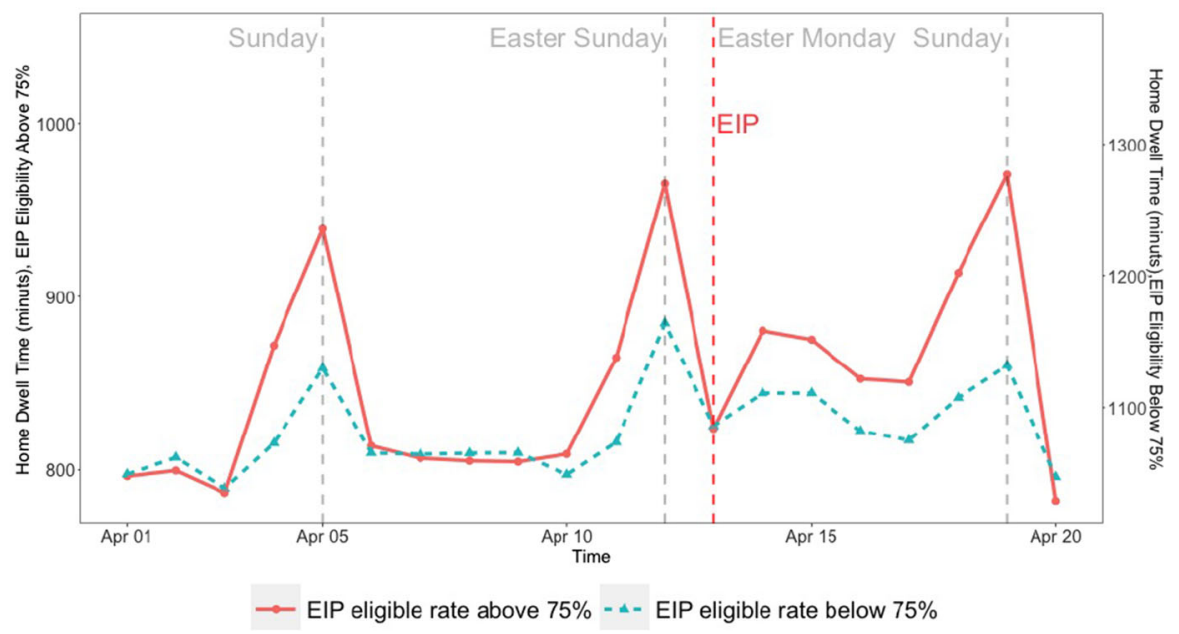

Fig. 3 Daily Median Home Dwell Time trends for CBGs with share of EIP reception more/less than 75\%

the county level because they are correlated with the time-varying individual preferences in mitigation efforts. $z_{i t}$ is a vector of three binary variables indicating different state-/county-level government policies. $\mu_{i}$ and $\tau_{t}$ are CBG and date fixed effects. $c_{i}$ is share of households eligible for receiving the EIP (income less than $\$ 150,000$ ). $I_{t=a}$ and $I_{c_{i}>75 \%}$ are indicator functions, which are equal to 1 if the conditions in the subscripts are satisfied and 0 otherwise. $d_{t}$ is a dummy variable with $d_{t}=0$ if date $t$ is a weekday and $d_{t}=1$ if date $t$ is a Saturday or Sunday. Since Figs. 2 and 3 suggest that the gap varies across weekdays and weekends regardless of the EIP treatment, $d_{t} \times I_{c_{i}>75 \%}$ captures that pre-existing variation. $\theta_{a}^{1}$ and $\theta_{a}^{2}$ capture the daily time trends of the low-income and high-income CBGs.

In addition, I compare the conditional daily gap in "Median Home Dwell Time" between the low-income and high-income CBGs as our the main analysis. Consider the following event study regression:

$$
\begin{aligned}
y_{i t}= & \sum_{a=-10}^{-1} \alpha_{a}^{\text {before }} I_{e_{i t}=a} \times I_{c_{i}>75 \%}+\sum_{a=1}^{8} \alpha_{a}^{\text {after }} I_{e_{i t}=a} \times I_{c_{i}>75 \%} \\
& +\beta x_{i t}+\rho z_{i t}+\lambda d_{t} \times I_{c_{i}>75 \%}+\mu_{i}+\tau_{t}+\epsilon_{i t},
\end{aligned}
$$

where $e_{i t}$ is the difference in days between date $t$ and April 12, 2020 (one day before receiving the EIP). $I_{e_{i t}=a}$ is the indicator function that equals 1 if the conditions in the subscripts are satisfied and 0 otherwise. $\alpha_{a}^{b e f o r e}$ and $\alpha_{a}^{a f t e r}$ capture the daily gap between the low-income and high-income CBGs. 


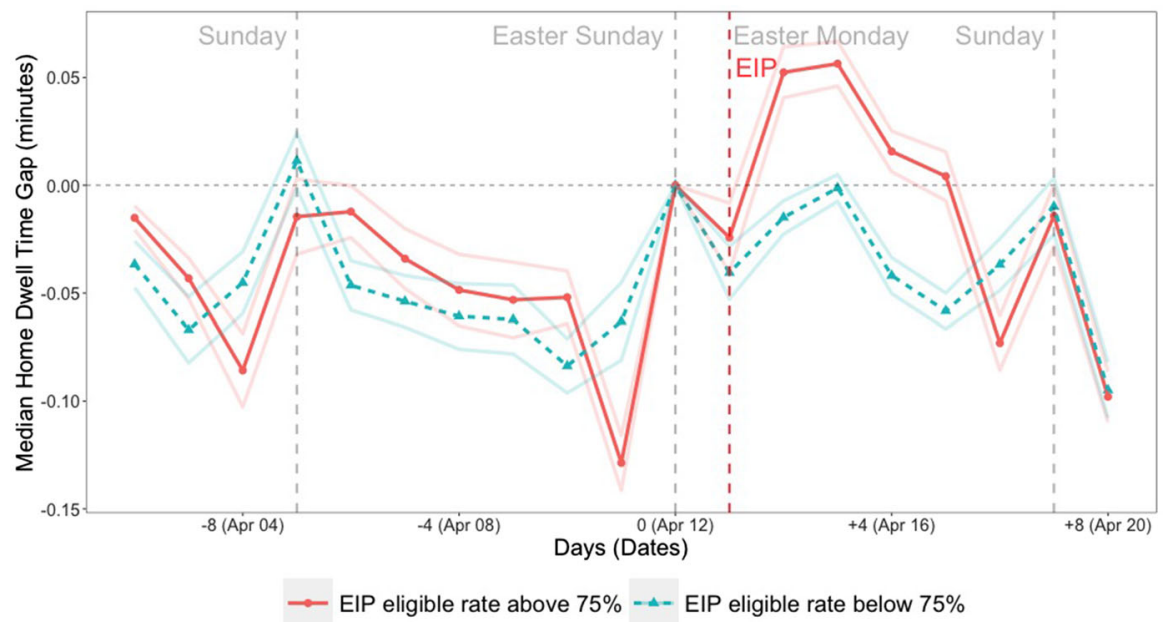

Fig. 4 Conditional daily trends, state policies

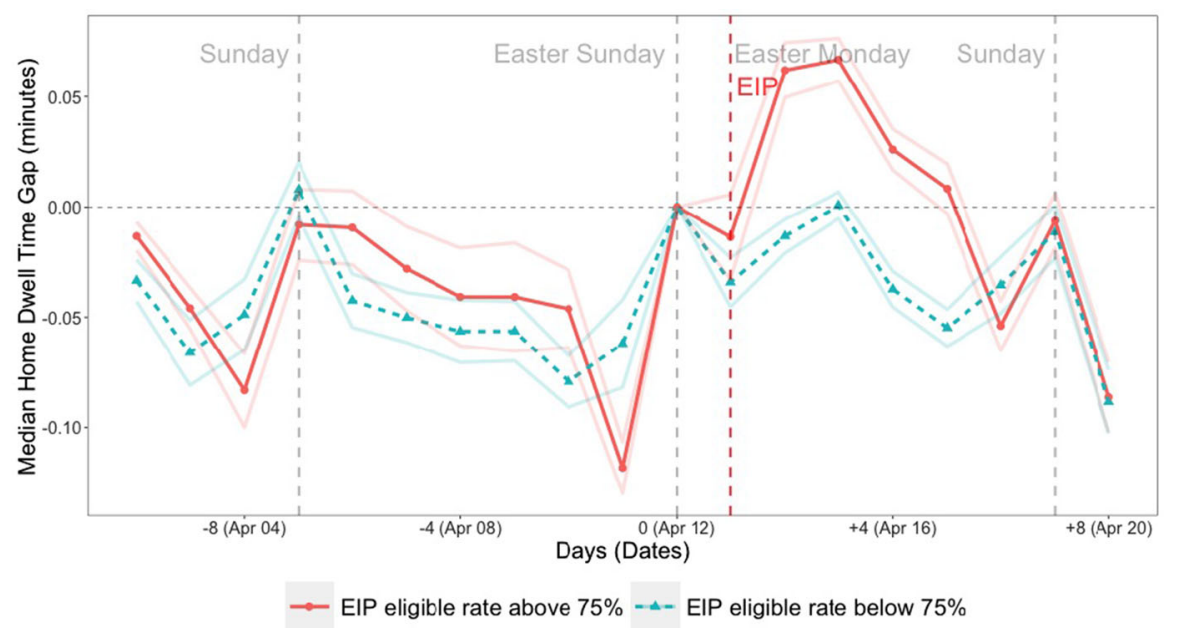

Fig. 5 Conditional daily trends, county policies

\subsection{Main results}

I first estimate the regression Eq. 1 using state/county-level policy data and plot the estimated daily trends of the low-income and high-income CBGs $\left(\theta_{a}^{1}\right.$ and $\left.\theta_{a}^{2}\right)$ in Figs. 4 and $5 .{ }^{12}$ There is a large difference in sample size between the state-level and county-level policy data: The state-level policy data contain 4,317,586 observations, whereas the county-level policy data only contain 2,541,943 observations. Despite the large difference in sample size, Figs. 4 and 5 show similar patterns: Before the

\footnotetext{
12 In the regression, I set April 12, 2020 as the reference day. The first day, April 1, 2020, is suppressed to capture the weekday/weekend differences between two groups of CBGs.
} 


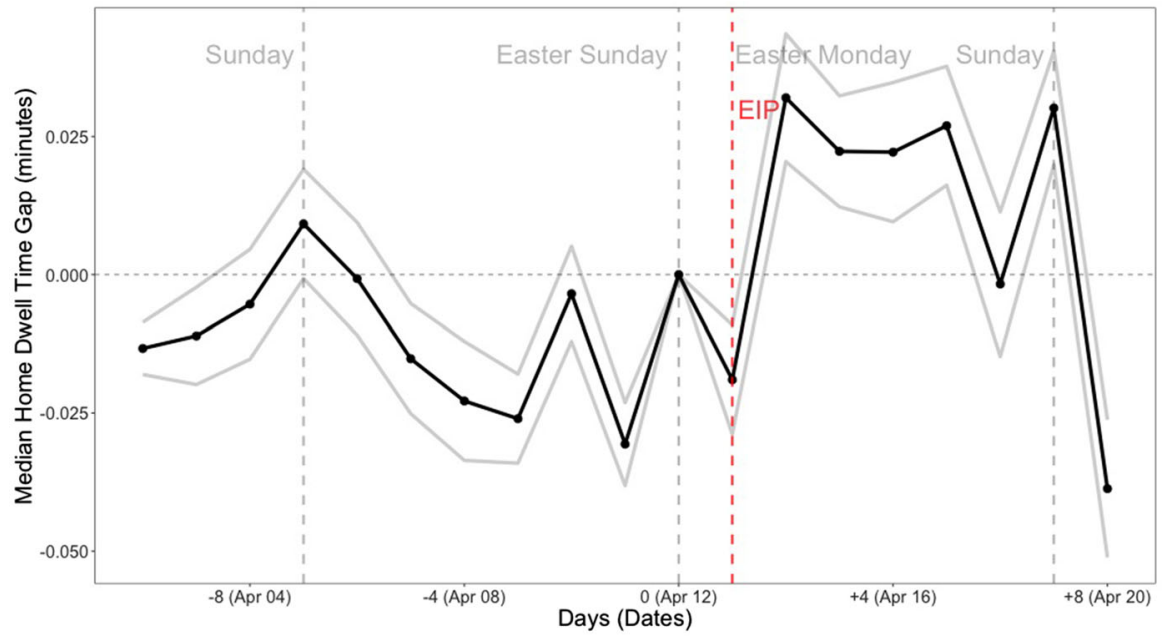

Fig. 6 Event study, state policies

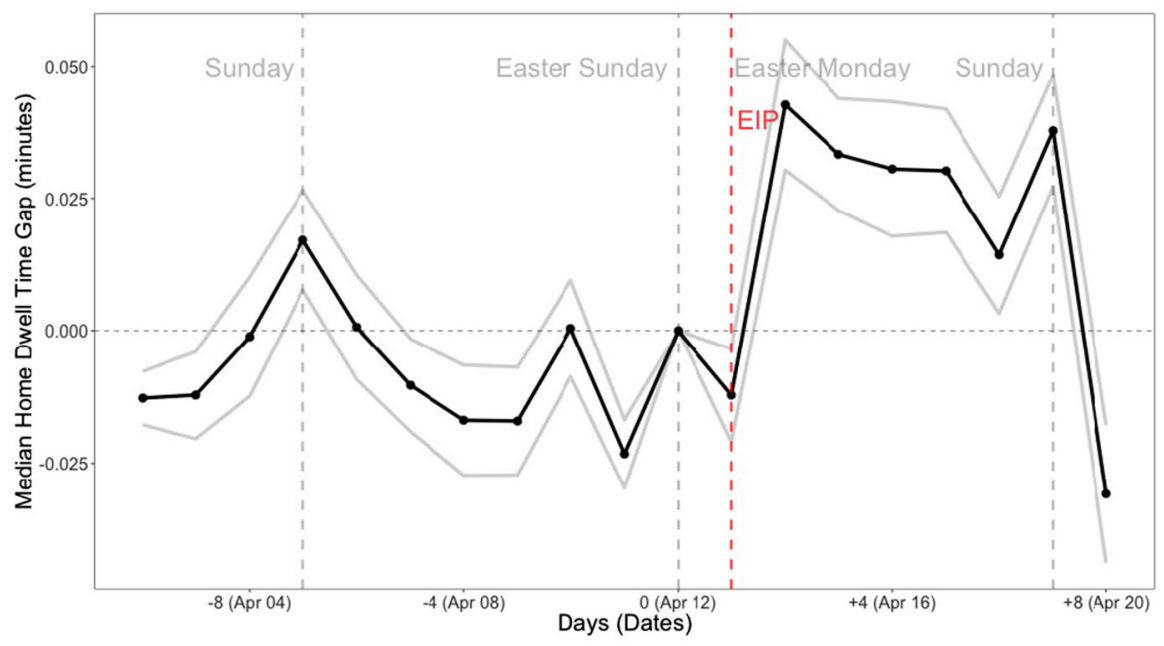

Fig. 7 Event study, county policies

EIP treatment, there is small difference in daily trends between the low-income and high-income CBGs; after the EIP treatment, there is a increase in daily trends for all CBGs, but the increase is much larger for the low-income CBGs than the high-income CBGs.

As the main analysis results, I then estimate the event study regression Eq. 2 and plot the estimated daily gaps $\alpha_{a}^{\text {before }}$ and $\alpha_{a}^{a f t e r}$. Figures 6 and 7 show the estimates of the regression using state-/county-level policy data, respectively. Again, both figures show similar patterns: Before the EIP treatment, the daily gaps are mostly negative; after the EIP treatment, there is a sharp increase in daily gaps, and such change persists 
for one week. These results are consistent with Figs. 4 and 5 and illustrate a causal relationship between the EIP treatment and COVID-19 mitigation efforts (human mobility), suggesting that, compared with the high-income CBGs, the low-income CBGs which received a larger size of the EIP treatment experienced a dramatic increase in "Median Home Dwell Time." These results are consistent with the main hypothesis that the EIP treatment decreases the human mobility.

In Online Appendix Table B1, I report the estimated results of the DID analysis. These results are consistent with the event study analysis, suggesting that the EIP treatment significantly increased "Median Home Dwell Time." In addition, the DID results also imply that, on average, the EIP treatment significantly increased "Median Home Dwell Time" by 3-5\% (about 26-45 min).

There may be some alternative mechanisms that can generate a positive EIP effect on "Median Home Dwell Time." For example, it may be caused by an increase in leisure due to income compensation. However, it is unlikely that the leisure can be affected in a 20-day period with a one-time $\$ 1200$ payment. Even if there is a leisure effect due to the EIP, it tends to appear in the relatively lower-income households, because the EIP raises their income in a relatively larger proportion. In the following section, I estimate the different EIP effects across incomes and show that the results indicate that the EIP has zero effect on low-income households, which suggests the results are not driven by the changes in leisure.

\section{Additional results and discussion}

\subsection{Subgroup differences in treatment effect}

Recall that the theory described in the previous section suggests that if the household incomes are lower than the income threshold, then they are "non-voluntary contributors" and contribute zero mitigation efforts. Under such circumstances, if the EIP amount is not large enough to convert them into "voluntary contributors," then the EIP has no effect on their mobility. In this section, I show that the EIP treatment effect may vary depending on income by analyzing the different EIP effects across the subgroups of households with different incomes. Since the income threshold is unknown, I analyze the subgroup treatment effects in a non-parametric approach by allowing the effects to be different across arbitrarily defined income subgroups.

To empirically identify the income threshold, I arbitrarily define several income subgroups and estimate the average treatment effect of each group in the DID framework (see Online Appendix B6). According to the results shown in Tables B2, B6, B7 and B8 in online appendix, the EIP treatment is insignificant for households with incomes less than $\$ 20,000$. This suggests that $\$ 0-20,000$ income subgroup is a good approximation for "non-voluntary contributors." With this prior, I divide the low-income CBGs into two subgroups: the "Voluntary Contributors' CBGs" and the "Non-voluntary Contributors' CBGs," depending on whether or not there was a majority of the households with incomes less than $\$ 20,000$ or not. Consider the following regression: 


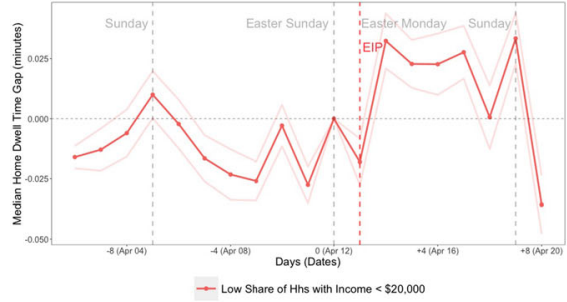

(a) "Voluntary Contributors' CBGs", less than half of the households have income lower than $\$ 20,000$

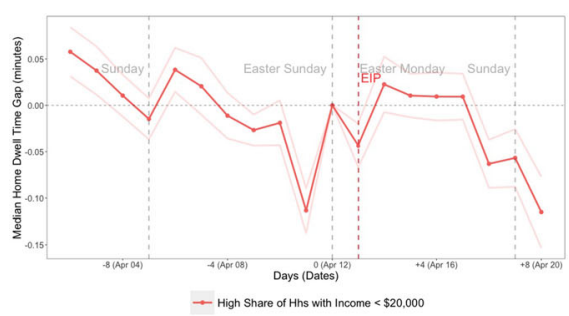

(b) "Non-voluntary Contributors' CBGs", more than half of the households have income lower than $\$ 20,000$

Fig. 8 Event study of subgroup (income) EIP effects, state policies

$$
\begin{aligned}
y_{i t}= & \sum_{a=-10}^{-1} \alpha_{a}^{1, \text { before }} I_{e_{i t}=a} \times I_{c_{i}>75 \%} \times I_{r_{i} \leq 50 \%} \\
& +\sum_{a=1}^{8} \alpha_{a}^{1, \text { after }} I_{e_{i t}=a} \times I_{c_{i}>75 \%} \times I_{r_{i} \leq 50 \%} \\
& +\sum_{a=-10}^{-1} \alpha_{a}^{2, \text { before }} I_{e_{i t}=a} \times I_{c_{i}>75 \%} \times I_{r_{i}>50 \%} \\
& +\sum_{a=1}^{8} \alpha_{a}^{2, \text { after }} I_{e_{i t}=a} \times I_{c_{i}>75 \%} \times I_{r_{i}>50 \%} \\
& +\beta x_{i t}+\rho z_{i t}+\lambda d_{t} \times I_{c_{i}>75 \%}+\mu_{i}+\tau_{t}+\epsilon_{i t},
\end{aligned}
$$

where $r_{i}$ is the share of households with income less than $\$ 20,000$ and $I_{r_{i} \leq 50 \%}$ and $I_{r_{i} \leq 50 \%}$ are indicator functions. $\alpha_{a}^{1, \text { before }}$ and $\alpha_{a}^{1, a f t e r}$ capture the daily gap between the high-income CBGs and the "Voluntary Contributors' CBGs"; $\alpha_{a}^{2, \text { before }}$ and $\alpha_{a}^{2, a f t e r}$ capture the daily gap between the high-income CBGs and the "Non-voluntary Contributors' CBGs." Using the state- and county-level policy data, Figs. 8a and 9a plot the estimated $\alpha_{a}^{1, \text { before }}$ and $\alpha_{a}^{1, a f t e r}$, Figs. 8b and $9 \mathrm{~b}$ plot the estimated $\alpha_{a}^{2, \text { before }}$ and $\alpha_{a}^{2, a f t e r}$. I find that the estimated daily gap between the high-income CBGs and the "Voluntary Contributors' CBGs" plotted in Figs. 8a and 9a follows a similar patterns to the main results shown in Figs. 6 and 7, suggesting that there is a strong EIP treatment effect on human mobility in the "Voluntary Contributors' CBGs." However, according to Figs. $8 \mathrm{~b}$ and $9 \mathrm{~b}$, there is no clear evidence that the EIP treatment affects human mobility in the "Non-voluntary Contributors' CBGs." These results are consistent with the results from the DID analysis and support the argument that "Income $\$ 0-20,000$ " is a good approximation for households who are "Non-voluntary Contributors."

There is a concern about the results of this subgroup analysis that relates to the timing of EIP receipt: All direct-deposit payments were received in the week of April 13, 2020, but for households without direct deposits, there was a time lag, with paper checks arriving by mail over subsequent weeks. My analysis only captures the EIP 


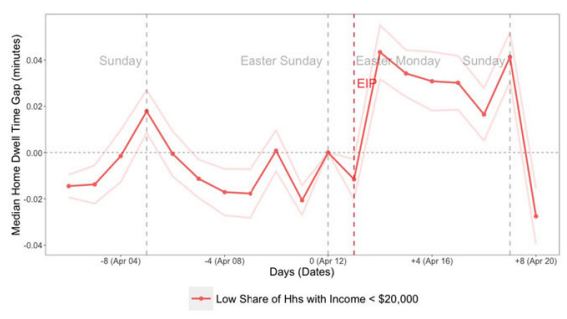

(a) "Voluntary Contributors' CBGs", less than half of the households have income lower than $\$ 20,000$

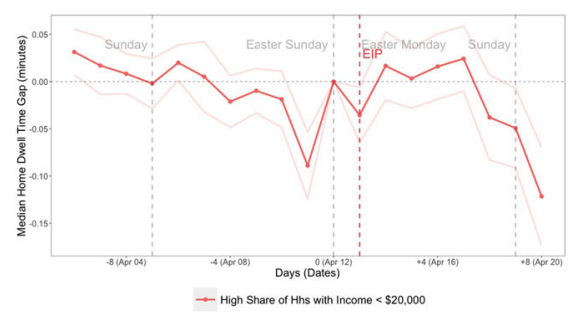

(b) "Non-voluntary Contributors' CBGs", more than half of the households have income lower than $\$ 20,000$

Fig. 9 Event study of subgroup (income) EIP effects, county policies

effect of the payments received through the direct deposits, so it is a lower bound of the true EIP effect on human mobility. If the low-income households are less likely to have the direct deposit accounts, that could also explain the null effect of EIP on these households. However, the estimates are reliable as long as the number of households without direct deposits does not dominate the whole population.

\subsection{Discussion}

An implicit assumption of this empirical analysis is that households' incomes (excluding EIP) remain fixed across the study period. This is a relatively strong assumption, since there is a large wave of lay-offs/furloughs during the pandemic. Although the day fixed effect captures the average income trend across all CBGs, with the possibility that lower-income workers are more likely to be laid off/furloughed, the variation in contemporary income shocks from unemployment (because of the different income distributions across the CBGs) may still cause failure to establish a causal relationship. I address this problem in two ways. First, I use a short study period between April 1, 2020, and April 20, 2020. According to BLS (2020) and Coibion et al. (2020), most of lay-offs occurred before the beginning of April, so the income shocks due to job loss are mild throughout the study period. ${ }^{13}$ Second, I conduct a robustness check analysis with a weaker time trend assumption, which includes state- and county-specific time fixed effects. These relaxed assumptions can partially capture the unobserved variation in income shocks from unemployment at the state and county levels. Therefore, if there are heterogeneous time trends across the states and counties due to unobserved variables, then they can be captured by the state- and county-specific time fixed effects. The results of this robustness check are reported in Fig. 10 for the event study analysis and panel II in Appendix Table B3 for the DID analysis. I find that the stateand county-specific time fixed effects did not change the results, suggesting that the assumption of constant income level (detrended by the day fixed effects) neither biases the estimated treatment effect nor does it violate the causal inference. In addition, the

13 BLS (2020) reported the April unemployment number was 23.1 million, Coibion et al. (2020) estimated that there were over 20 million unemployment at the beginning of April. These numbers suggest that the major unemployment wave occurred before the beginning of April, and unemployment rate was relatively stable within the month of April. 


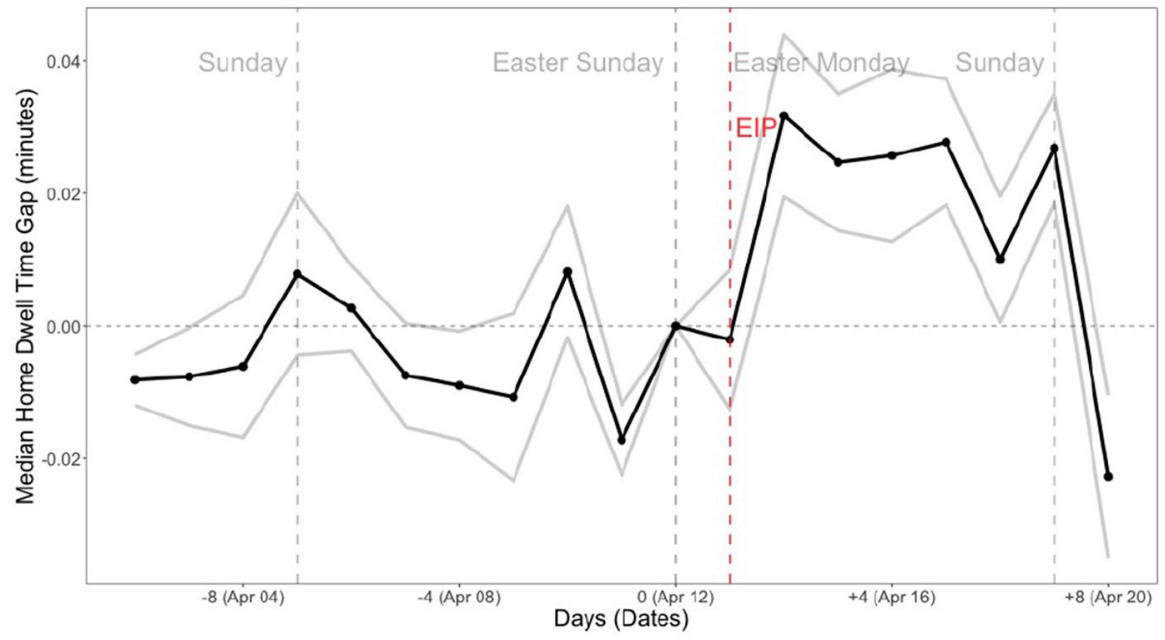

(a) Event Study, State Policies and State-Specific Time Trend

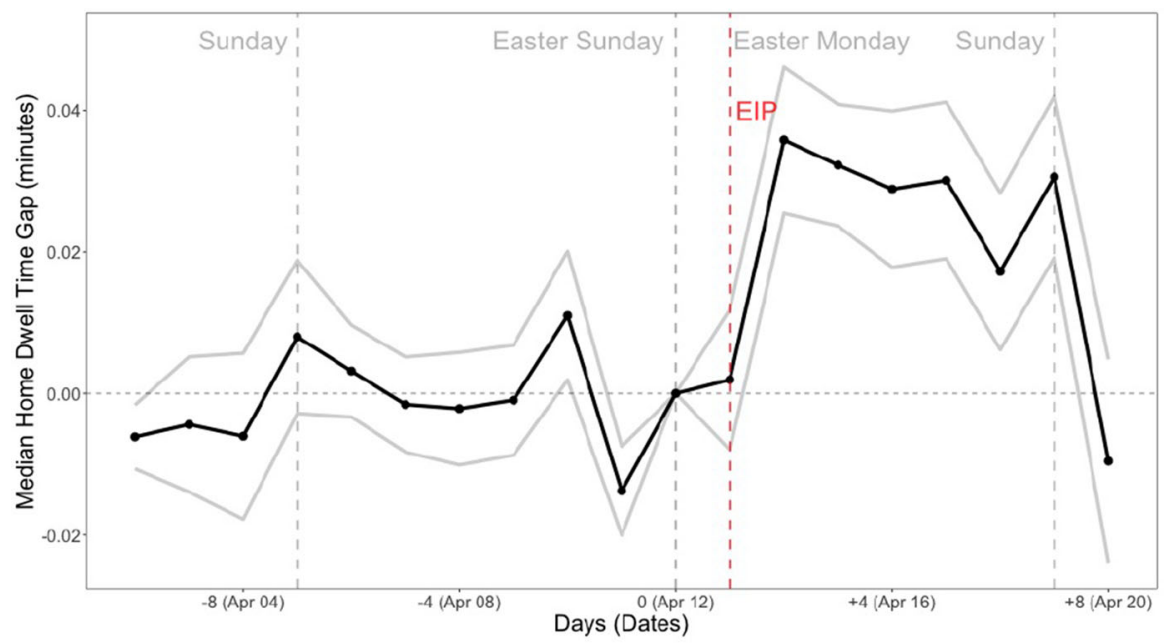

(b) Event Study, County Policies and County-Specific Time Trend

Fig. 10 Event study with state-/county-specific time trend

more relaxed time trend assumptions seem helpful in controlling some unobserved variations, so that it improves the parallel trends in the pre-treatment period with a much smaller number of significantly nonzero estimates of daily coefficients.

\section{Conclusion}

During the COIVD-19 pandemic in early 2020, mitigating the virus spreading heavily depended on every individual's contributions to mitigation efforts. To increase 
the individual and social aggregate mitigation efforts, a common government action is to impose mandatory policies that set a minimum requirement for the mitigation efforts of all individuals. However, in many countries including the USA, due to a variety of economic, social and political issues, it is difficult to strictly enforce such mandatory policies. In this paper, I propose that other than mandatory policies, income compensation policy is an alternative government instrument, which provides a possible decentralized solution to increase the individual and social aggregate mitigation efforts.

To empirically test whether income compensation increases individual contributions to mitigation efforts, I use the first round of EIP in the USA as a quasi-natural experiment and study the effect of receiving the EIP on human mobility ("Median Home Dwell Time"), where human mobility is an indicator of contributions to the COVID-19 mitigation efforts. The empirical results show that the EIP significantly increased "Median Home Dwell Time" by 3\%-5\% on average, though this increase was driven primarily by households with incomes greater than $\$ 20,000$. For households with incomes below $\$ 20,000$, the EIP amount distributed at the time was insufficient to motivate individuals/households to increase their contributions to the COVID-19 mitigation efforts.

Through exploring the public goods nature of the COVID-19 mitigation efforts, this paper highlights an unintended benefit of the EIP: In addition to providing economic assistance during the pandemic, the EIP also motivated households to increase their contributions to the COVID-19 mitigation efforts. However, there are several limitations in this study that need to be addressed by future research. First, due to a lack of data, the current empirical analysis is at the CBG-aggregate level instead of the individual or household level. Second, due to the limited access to SafeGraph human mobility data, the data period in this paper are restricted to the year 2020. Since almost all CBGs received the EIP, the restricted sample period makes the control group unavailable to the analysis. Further studies would benefit from collecting the data for previous years to use as as the control group in order to conduct a cross-year analysis.

Supplementary Information The online version contains supplementary material available at https://doi. org/10.1007/s00181-021-02117-0.

\section{Declarations}

Conflict of interest Ruohao Zhang declares that he has no conflict of interest.

Ethical approval This article does not contain any studies with human participants or animals performed by any of the authors.

\section{References}

Acemoglu D, Finkelstein A, Notowidigdo MJ (2013) Income and health spending: evidence from oil price shocks. Rev Econ Stat 95(4):1079-1095

Allcott H, Boxell L, Conway J, Gentzkow M, Thaler M, Yang D (2020) Polarization and public health: partisan differences in social distancing during the coronavirus pandemic. J Public Econ 191:104-254 
Bai J, Du S, Jin W, Wan C (2020) The impact of social capital on individual responses to COVID-19 pandemic: evidence from social distancing. In: SSRN working paper 3609001

Barrios JM, Benmelech E, Hochberg YV, Sapienza P, Zingales L (2021) Civic capital and social distancing during the COVID-19 pandemic. J Public Econ 193:104310

BLS (2020). News release: the unemployment situation. In: Bureau of labor statistics, May 8th, 2020

Buckley E, Croson R (2006) Income and wealth heterogeneity in the voluntary provision of linear public goods. J Public Econ 90(1):935-955

Chudik A, Pesaran M, Rebucci A (2020) Voluntary and mandatory social distancing: evidence on COVID-19 exposure rates from Chinese provinces and selected countries. In: NBER working paper 27039

Coibion O, Gorodnichenko Y, Weber M (2020) Labor markets during the COVID-19 crisis: a preliminary view. In: NBER working paper 27017

Dave DM, Friedson AI, Matsuzawa K, Sabia JJ (2020) When do shelter-in-place orders fight COVID-19 best? Policy heterogeneity across states and adoption time. Econ Inq 59(1):29-52

Durante R, Guiso L, Gulino G (2021) Asocial capital: Civic culture and social distancing during COVID-19. J Public Econ 194(1):104342

Evans WN, Moore TJ (2011) The short-term mortality consequences of income receipt. J Public Econ 95(11):1410-1424

Farboodi M, Jarosch G, Shimer R (2021) Internal and external effects of social distancing in a pandemic. J Econ Theory (forthcoming)

Fowler JH, Hill SJ, Obradovich N, Levin R (2021) Stay-at-home orders associate with subsequent decreases in COVID-19 cases and fatalities in the United States. PLoS ONE 16(6):e0248849

Gollwitzer A, Martel C, Brady WJ, Pärnamets P, Freedman IG, Knowles ED, Van Bavel JJ (2020) Partisan differences in physical distancing are linked to health outcomes during the COVID-19 pandemic. Nat Hum Behav 4(11):1186-1197

Gross T, Tobacman J (2014) Dangerous liquidity and the demand for health care: evidence from the 2008 stimulus payments. J Hum Resour 49(2):424-445

Gupta S, Rojas FL, Raman S, Lee B, Bento A, Simon KI, Wing C (2020) Tracking public and private responses to the COVID-19 epidemic: evidence from state and local government actions. In: NBER working paper 27027

Jacobsen GD, Kotchen MJ, Vandenbergh MP (2012) The behavioral response to voluntary provision of an environmental public good: evidence from residential energy demand. Eur Econ Rev 56(1):946-960

Johnson DS, Parker JA, Souleles NS (2006) Household expenditure and the income tax rebates of 2001. Am Econ Rev 96(5):1589-1610

Kapoor R, Rho H, Sangha K, Sharma B, Shenoy A, Xu G (2020) God is in the rain: the impact of rainfallinduced early social distancing on COVID-19 outbreaks. In: SSRN working paper 3605549

Kotchen MJ, Moore MR (2007) Private provision of environmental public goods: Household participation in green-electricity programs. J Environ Econ Manag 53(1):1-16

Mangrum D, Niekamp P (2020) JUE Insight: College student contribution to local COVID-19 spread: evidence from university spring break timing. J Urban Econ Insight, forthcoming

Wright AL, Sonin K, Driscoll J, Wilson J (2020) Poverty and economic dislocation reduce compliance with COVID-19 shelter-in-place protocols. J Econ Behav Organ 180:544-554

Publisher's Note Springer Nature remains neutral with regard to jurisdictional claims in published maps and institutional affiliations. 